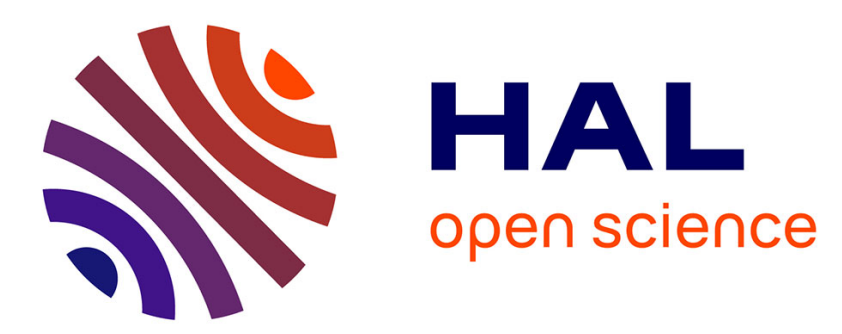

\title{
Reduction of Bloch-Floquet bending waves via annular acoustic black holes in periodically supported cylindrical shell structures
}

Jie Deng, Oriol Guasch, Laurent Maxit, Ling Zheng

\section{- To cite this version:}

Jie Deng, Oriol Guasch, Laurent Maxit, Ling Zheng. Reduction of Bloch-Floquet bending waves via annular acoustic black holes in periodically supported cylindrical shell structures. Applied Acoustics, 2020, 169, pp.107424. 10.1016/j.apacoust.2020.107424 . hal-02952063

\section{HAL Id: hal-02952063 \\ https://hal.science/hal-02952063}

Submitted on 29 Sep 2020

HAL is a multi-disciplinary open access archive for the deposit and dissemination of scientific research documents, whether they are published or not. The documents may come from teaching and research institutions in France or abroad, or from public or private research centers.
L'archive ouverte pluridisciplinaire HAL, est destinée au dépôt et à la diffusion de documents scientifiques de niveau recherche, publiés ou non, émanant des établissements d'enseignement et de recherche français ou étrangers, des laboratoires publics ou privés. 


\title{
Reduction of Bloch-Floquet bending waves via annular acoustic black holes in periodically supported cylindrical shell structures
}

\author{
Jie Deng ${ }^{\mathrm{a}, \mathrm{b}}$, Oriol Guasch ${ }^{\mathrm{b}, *}$, Laurent Maxit ${ }^{\mathrm{c}}$, Ling Zheng ${ }^{\mathrm{a}}$ \\ a State Key Laboratory of Mechanical Transmission, College of Automotive Engineering \\ Chongqing University, Chongqing 400044, PR China \\ ${ }^{b}$ GTM - Grup de Recerca en Tecnologies Mèdia, La Salle, Universitat Ramon Llull \\ C/ Quatre Camins 30, 08022 Barcelona, Catalonia (Spain) \\ ${ }^{c}$ INSA-Lyon, Laboratoire Vibrations-Acoustique (LVA) \\ 25 bis, av. Jean Capelle, F-69621 Villeurbanne Cedex, France
}

\begin{abstract}
In recent years, acoustic black holes (ABHs) have revealed as a very effective method for the passive reduction of vibrations in flat plates and straight beams. Those essentially consist of circular indentations and/or side boundaries, whose thicknesses decrease to zero following a power-law profile. Nonetheless, many structures in the aeronautical and naval sectors, among others, involve cylindrical structures with periodic stiffeners or supports. Such periodic structures admit the propagation of Bloch-Floquet (BF) bending waves at some frequency passbands, which may result in strong vibration levels and noise radiation. In this work, we propose the design of annular ABHs to mitigate the problem. A wave finite element model is first used to determine the frequency passbands on an ideal, periodically simply supported cylindrical shell of infinite length, with embedded annular ABHs. Then, a long, but now finite simply supported cylindrical shell, is presented to show how the transmissibility between two sections strongly diminishes with the inclusion of ABHs. That confirms annular ABHs as a very effective means of reducing BF wave propagation. Unfortunately, embedding ABHs on the shell weakens its structural rigidity so a thorough analysis is made on the effects of inserting stiffeners in the longitudinal direction to partially remedy that inconvenient. Quite surprisingly, it is shown that the inclusion of stiffeners can even enhance the performance of the ABHs for some configurations. To finally foresee the potential of annular $\mathrm{ABHs}$ for practical applications, a finite element simulation is made on a slightly more realistic geometry, resembling a very simplified version of the hull of a submarine vehicle or a gas tank.
\end{abstract}

Keywords: Annular acoustic black holes (ABHs), Periodic structures, Bloch-Floquet waves, Stiffeners, Cylindrical shells

\section{Introduction}

The vibration of structures with periodic supports or stiffeners has now a long record since the earlier research by Mead in the seventies (see e.g., [1-3] and also the review in [4]). Those works revealed, for instance, that in a periodically simply supported infinite beam flexural wave propagation can only occur at certain frequency passbands, while it is forbidden for other frequency ranges, the so-called stopbands. Similar results were lately found for infinite periodically stiffened plates, see e.g., [5-8]. It is to be noted that propagative waves at the passbands of periodically stiffened systems, also referred to as Bloch-Floquet (BF) waves, often constitute a major source of undesirable vibration and noise radiation.

In this paper, we are concerned with periodically supported cylindrical structures. Those are of great importance in a large variety of industrial applications which comprise from aircrafts and submarines, to

*Corresponding Author: oriol.guasch@salle.url.edu 
pipeline systems and gas/fluid tanks. Despite a large amount of literature exists on the behavior of periodic stiffened beams and plates, not so many works can be found characterizing the dynamical response of periodically stiffened cylindrical shells. Initial analyzes were reported in [9-11]. Exact-closed solutions were proposed therein for some periodically ribbed cylindrical shells with various stiffener geometries, and the frequency passbands and stopbands of the structures were determined. Following the steps in [12], more recent works on the topic have basically addressed the computation of noise radiation by periodic cylindrical shells under different types of excitations (see e.g., [13-17]), yet such issue will be out of the scope of this paper.

The main objective of this work is that of exploring, by means of numerical simulations, the potential of the acoustic black hole $(\mathrm{ABH})$ effect as a passive method to reduce the propagation of BF waves in periodically simply supported cylindrical shells. To that purpose, new annular ABH designs are proposed and their performance is tested by means of the wave finite element method [18]. The ABH effect in mechanics was discovered by Mironov [19], who showed that the velocity of a wave entering a beam termination whose thickness decays to zero following a power-law profile, it would slow down in such a way that it will never reach the end of the wedge. In practice however, even a small truncation thickness at the wedge termination may result in strong wave reflections. The problem can be avoided by placing a viscoelastic layer at the tip of the wedge to dissipate energy where it most concentrates [20]. Recently, several strategies have been investigated to enhance energy dissipation and thus reduce the $\mathrm{ABH}$ reflection coefficient, such as setting an extended platform at the end of the $\mathrm{ABH}$ wedge [21, 22], exploiting the $\mathrm{ABH}$ geometric non-linearity [23], using spiral terminations [24, 25], resorting to passive constrained viscoelastic layers [26] or employing viscoelastic layers with tunable parameters [27].

When one considers beams and plates belonging to a more complex structure, however, it becomes unfeasible to tailor their sides to attain the $\mathrm{ABH}$ effect, because the connection with other structural elements usually takes place through boundaries. To avoid that problem in a plate, for instance, one can embed circular cuneate indentations in it whose thicknesses decrease towards their center following a power law profile [28-33]. Though less common, rectangular ABH indentations have been also analyzed in [34, 35], as well as the performance of circular ABHs on elliptical instead of rectangular plates [36]. Very recently, further configurations have been examined, like the ring-shaped ABHs for vibration isolation in plates in [37], or the $\mathrm{ABH}$ indentations for circular beams in [38]. This last work addresses, for the first time, embedding ABHs on curved structures. The current paper makes progress in this direction by proposing suitable annular $\mathrm{ABH}$ designs for cylindrical shell structures. As said above, such issue has not been addressed before and constitutes the key goal of this research, a preliminary version of which was presented in [39].

The main contents of the manuscript can be outlined as follows. We start analyzing the case of an infinite cylindrical shell of uniform thickness that has periodic simple supports and determine its frequency passbands and stopbands. Then, a finite model consisting of a sample of ten unit cells of the infinite shell is used to compute the transmissibility between cell rings and observe the influence of the passbands and stopbands. The same configurations are next analyzed but with annular ABHs embedded on them. It is shown that the inclusion of ABHs strongly reduce the propagation of BF waves resulting in much lower transmissibilities on the shell. Besides, given that embedding $\mathrm{ABH}$ indentations on a structure affects its rigidity, we investigate the influence of inserting stiffeners on the annular ABHs. Finally, the benefits of setting $\mathrm{ABH}$ s on a periodically supported cylindrical shell with closed ends are described. That closed geometry could be viewed, for example, as a very simple representation of the hull of a submarine vehicle or an airplane fuselage. All the analyzes in the manuscript have been carried out by means of finite element simulations, as done in some previous works on ABHs, see e.g., [28] among others.

In what concerns periodic distributions of $\mathrm{ABHs}$, it is worth mentioning that they have been recently studied for arrangements of circular ABH indentations on beams and plates in [38, 40, 41]. New designs involving compound $\mathrm{ABH}$ beams [42] and V-folded ones [43, 44] have been also proposed. On the other hand, it is shall be noted that the $\mathrm{ABH}$ effect is not only useful for reducing vibrations in beams, plates or shells as just explained, but the very same basic underlying mechanisms can be exploited to attenuate sound waves at the end of duct terminations [45-49].

This paper is organized as follows. In Section 2 we present the finite element models of the considered cylindrical shells, as well as a brief theoretical overview on the wave finite element model for periodic 

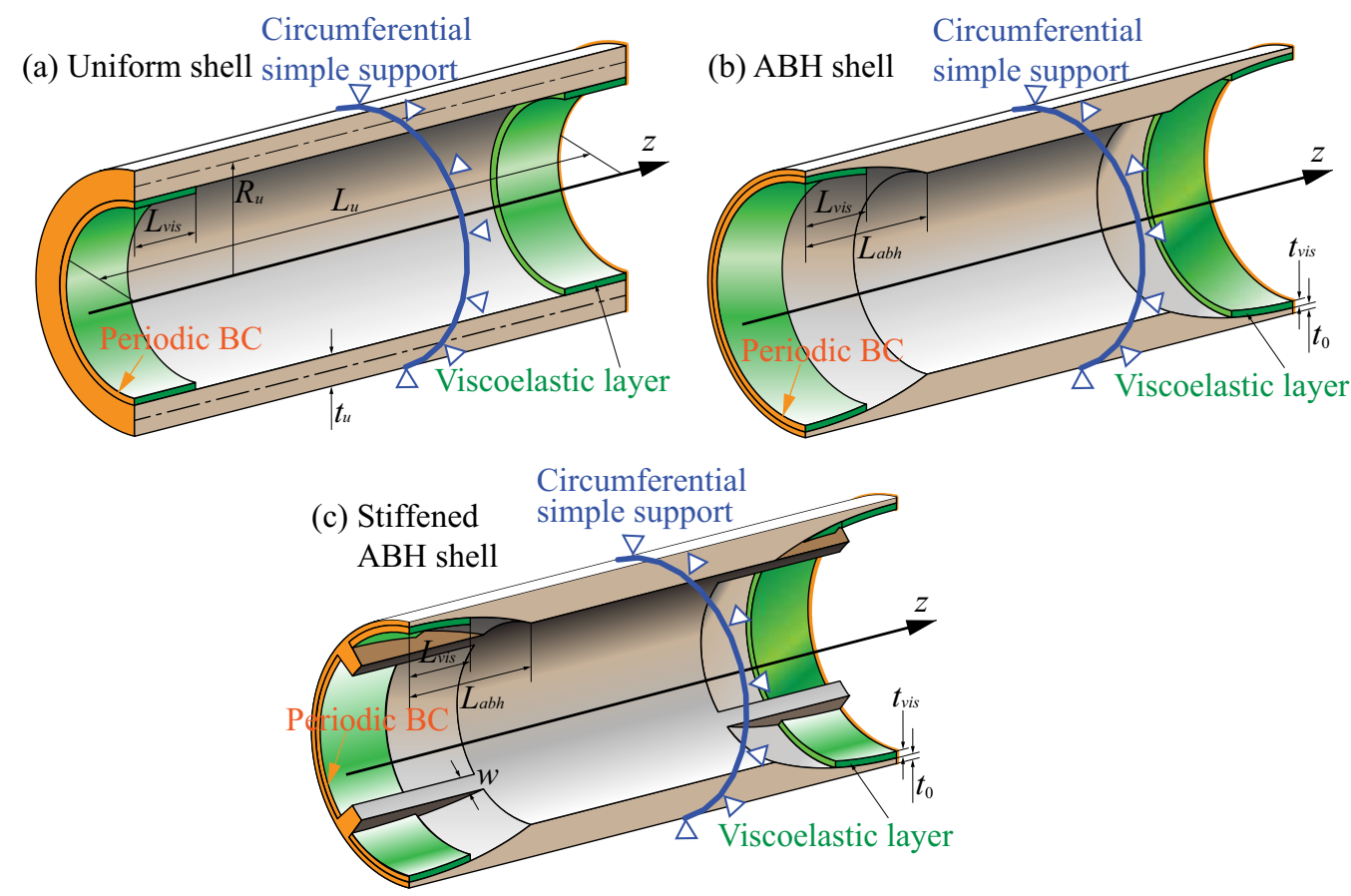

Figure 1: Unit cell for (a) the uniform cylindrical shell, (b) the ABH cylindrical shell and (c) the ABH cylindrical shell with reinforcing stiffeners.

structures. Section 3 constitutes the main body of the work and shows the dynamic responses of the infinite and finite shell models. The results include the analysis of frequency passbands and stopbands, transmissibility functions. The role of stiffeners and the case of a shell with closed ends are studied in Section 4. The final conclusions close the paper in Section 5.

\section{Finite element models}

\subsection{Infinite periodically supported cylindrical shell with and without annular ABHs}

\subsubsection{Model description}

To start analyzing the performance of annular ABHs on cylindrical shells we address the case of an infinite cylindrical shell with periodic simple supports. In fact, two infinite shells will be considered, a first one having uniform thickness and a second one with annular ABHs embedded in it. These models will serve to analyze the frequency passbands and stopbands of the structure, and check the influence of the ABHs on them.

In Fig.1, we depict the sketches of the unit cells that will serve to characterize the infinite cylindrical shells. Fig.1a corresponds to that with uniform thickness. As observed, the unit cell is simply supported at its center ring, having periodic boundary conditions at both ends. It has radius $R_{u}$, length $L_{u}$ and thickness $t_{u}$. Besides, in Fig.1b, we show the unit cell with an embedded annular ABH. The cell is also simply supported at its center with again periodic conditions at both ends. The ABH indentation comprises a portion $L_{\mathrm{abh}}$ of $L_{u}$ and reaches its thinner portion at the boundaries, which have residual thickness $t_{0}$. The local thickness inside the $\mathrm{ABH}$ varies as $t(z)=\varepsilon\left|z-z_{0}\right|^{m}+t_{0}$ where $z_{0}$ represents the location of the ABH center, $m(m \geq 2)$ is the ABH order and $\varepsilon=\left(t_{u}-t_{0}\right) L_{\text {abh }}^{-m}$ is the smoothness parameter that describes the $\mathrm{ABH}$ profile. A centered viscoelastic layer of thickness $t_{\mathrm{vis}}$, which covers $50 \%$ of the $\mathrm{ABH}$ surface, has been glued to the shell to dissipate energy. The same amount of damping has been applied to the uniform cylinder for a fairer comparison of the results, as observed in Figs.1a and 1b. Finally, in Fig.1c we plot the 
case of an $\mathrm{ABH}$ cell with some reinforcing stiffeners to try to compensate the loss of rigidity produced by the ABH indentations. Such situation will be also analyzed in this work.

Finite element models have been built for the uniform and ABH unit cells (with and without stiffeners), considering the geometrical and physical parameters of Table 1. The commercial package Comsol Multiphysics has been adopted for that purpose using the Solid Mechanics (2D axisymmetric) package. Quadrilateral shell elements have been employed. For the uniform shell, the model consists of 100 elements and 184 nodes for a total of 954 degrees of freedom (DOF). The mesh size, $h_{u}$, ranges between $0.0135 \mathrm{~m}<h_{u}<0.0169 \mathrm{~m}$, which guarantees wave propagation is well recovered up to $11.2 \mathrm{kHz}$. A somewhat finer mesh has been required for the ABH shell because of its small truncation thickness. The ABH unit cell FEM model consists of 160 elements, 284 nodes and 1454 DOF, with a mesh size comprising $0.003 \mathrm{~m}<h_{\mathrm{abh}}<0.0169 \mathrm{~m}$. That allows one to cover the same frequency range of the uniform shell.

\subsubsection{Theory reminder}

For a better comprehension of the results of the numerical simulations in Section 3 below, and to make the material in this work more self-contained, we next briefly review the basics of the Bloch-Floquet theory, which will lead to the appearance of frequency stopbands and passbands for the cylinder flexural waves.

Let us denote by $\mathbf{M}_{c}$ the condensed FEM mass matrix for the unit cell and by $\mathbf{K}_{c}$ the condensed stiffness one. These matrices relate the vectors of nodal displacements at the left and right sides of the cell, respectively denoted by $\mathbf{q}_{L}$ and $\mathbf{q}_{R}$. Hereafter, $\mathbf{M}_{c}$ and $\mathbf{K}_{c}$ can either designate the unit cell condensed matrices for the uniform shell or that for the $\mathrm{ABH}$ one, as the general theory applies to both of them. The same is valid for $\mathbf{q}_{L}$ and $\mathbf{q}_{R}$. Note that the condensed mass and stiffness matrices also incorporate the damping layers, which are critical for the $\mathrm{ABH}$ effect.

At a given angular frequency, $\omega$, the equation of motion for the cell boundary nodes reads (see e.g., $[50,51])$,

$$
\left(\mathbf{K}_{c}-\omega^{2} \mathbf{M}_{c}\right) \mathbf{q}=\mathbf{f},
$$

with $\mathbf{q}=\left[\begin{array}{l}\mathbf{q}_{L}^{\top} \mathbf{q}_{R}^{\top}\end{array}\right]^{\top}$ and $\mathbf{f}=\left[\mathbf{f}_{L}^{\top} \mathbf{f}_{R}^{\top}\right]^{\top}$ being the nodal force vector. The periodic boundary conditions and force balance imply

$$
\mathbf{q}_{R}=\lambda \mathbf{q}_{L}, \quad \mathbf{f}_{R}=-\lambda \mathbf{f}_{L},
$$

where $\lambda=\exp \left(-\mathrm{i} k L_{u}\right)$ and $k \in \mathbb{R}$ represents the propagating wave number. This leads to

$$
\mathbf{q}=\boldsymbol{\Lambda}_{R} \mathbf{q}_{L}, \quad \boldsymbol{\Lambda}_{L} \mathbf{f}=\mathbf{0},
$$

\begin{tabular}{ccc}
\hline Parameter & Value & Description. \\
\hline$R_{u}$ & $5 \mathrm{~m}$ & Radius of cylindrical shells \\
$t_{u}$ & $0.03 \mathrm{~m}$ & Thickness of uniform shell \\
$t_{0}$ & $0.003 \mathrm{~m}$ & Residual thickness of the ABH center \\
$L_{u}$ & $1.35 \mathrm{~m}$ & Length of one cell \\
$L_{\mathrm{abh}}$ & $0.325 \mathrm{~m}$ & Length of the ABH portion \\
$m$ & 2 & Order of ABH profile \\
$\varepsilon$ & $0.19231 \mathrm{1} / \mathrm{m}$ & Slope of ABH profile \\
$L_{\mathrm{vis}}$ & $0.1625 \mathrm{~m}$ & Length of viscoelastic layers \\
$t_{\text {vis }}$ & $0.006 \mathrm{~m}$ & Thickness of viscoelastic layers \\
$E$ & $210 \mathrm{GPa}$ & Young modulus of the shell \\
$\rho$ & $7800 \mathrm{~kg} / \mathrm{m}^{3}$ & Density of the shell \\
$\eta$ & 0.005 & Steel loss factor \\
$E_{\text {vis }}$ & $5 \mathrm{GPa}$ & Young modulus of viscoelastic layers \\
$\rho_{\text {vis }}$ & $950 \mathrm{~kg} / \mathrm{m}^{3}$ & Density of viscoelastic layers \\
$\eta_{\text {vis }}$ & 0.5 & Loss factor of viscoelastic layers \\
\hline
\end{tabular}

Table 1: Geometry and material parameters of the annular ABH shells. 


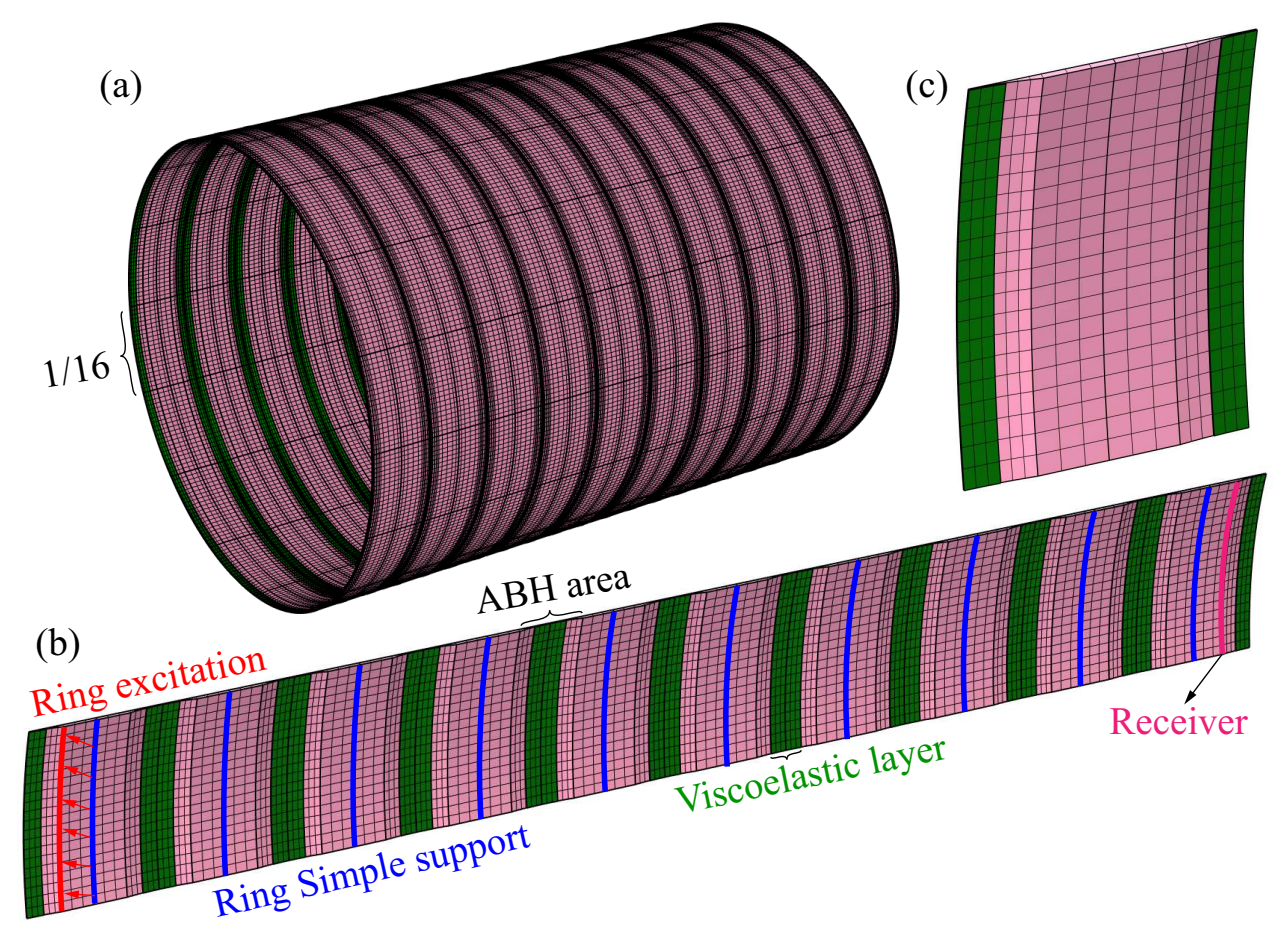

Figure 2: Finite element geometry and mesh details for the ten-cell cylindrical shell. a) 3D view, b) 1/16 longitudinal section with the location of the excitation, ABHs, boundary conditions and ring receiver and c) zoom of $1 / 16$ of a single cell.

with $\boldsymbol{\Lambda}_{R}=[\mathbf{I} \lambda \mathbf{I}]^{\top}$ and $\boldsymbol{\Lambda}_{L}=\left[\mathbf{I} \lambda^{-1} \mathbf{I}\right]$ (here $\mathbf{I}$ symbolizes the identity matrix of size $1 / 4$ that of the condensed mass matrix $\mathbf{M}_{c}$ ). Pre-multiplying both sides of Eq. (1) by $\boldsymbol{\Lambda}_{L}$ and making use of Eq. (3), results in

$$
\boldsymbol{\Lambda}_{L}\left(\mathbf{K}_{c}-\omega^{2} \mathbf{M}_{c}\right) \boldsymbol{\Lambda}_{R} \mathbf{q}_{L}=\mathbf{0},
$$

which yields an eigenvalue problem for the nodal displacements of the unit cell left side, namely,

$$
\left[\mathbf{K}_{r}(k)-\omega^{2} \mathbf{M}_{r}(k)\right] \mathbf{q}_{L}=\mathbf{0} .
$$

In Eq. (5), $\mathbf{K}_{r}(k)=\boldsymbol{\Lambda}_{L} \mathbf{K}_{c} \boldsymbol{\Lambda}_{R}$ and $\mathbf{M}_{r}(k)=\boldsymbol{\Lambda}_{L} \mathbf{M}_{c} \boldsymbol{\Lambda}_{R}$ respectively denote the reduced stiffness and mass matrices. The solution of Eq. (5) for $k$ in the range $[-\pi / L \pi / L]$ (i.e., in the first Brillouin zone) provides the dispersion relationship for the the periodically simply supported uniform and ABH cylindrical shells. Thanks to the unit cell symmetry, it would actually suffice to consider the range $[0 \pi / L]$.

The WFEM method has been only succinctly reviewed in this section, which suffices for the purposes of this paper. The interested reader is referred to [52-54] for more details and explanations.

\subsection{Finite periodically supported cylindrical shell with and without annular ABHs}

\subsubsection{Model description}

In order to check whether annular ABHs could have a significant effect on more realistic structures, we also consider the case of a finite cylindrical shell consisting of ten, equally spaced, concatenated unit cells. A simply supported ring is imposed in the middle of each cell, while free displacement and rotation conditions are assumed at the leading ends of the structure. The cylinder longitudinal dimension is $L=13.5 \mathrm{~m}$ so that each unit cell has a length of $L_{u}=1.35 \mathrm{~m}$ to coincide with that of the infinite structure. The radii, thicknesses and all other geometrical and physical parameters, including damping layers, for both the uniform finite shell and the ABH one, are those already chosen for the infinite structures and presented in Table 1. 
The uniform and ABH cylindrical ten-cell shells have been simulated again with FEM. While a 2D axis symmetric package was used in the infinite elements models of section 2.1, the 3D Solid Mechanics package in Comsol Multiphysics has been chosen for building the finite ones below. This is so because in these models we will consider the inclusion of stiffeners. However, to reduce the computational cost, and thanks to the problem symmetry, only $1 / 16$ of the whole $3 \mathrm{D}$ structure has been modeled (see Fig. 2 for the ABH one). In this case, the FEM model consists of 54069 DOF with a total of 18240 hexahedron elements and 32152 nodes for the uniform shell, while 20280 hexahedron elements and 36075 nodes have been used for the $\mathrm{ABH}$ one. The mesh size for the latter, $h_{\mathrm{abh}}$, comprises $0.003 \mathrm{~m}<h_{\mathrm{abh}}<0.05625 \mathrm{~m}$ and covers the frequency up to $5.8 \mathrm{kHz}$. The latter also holds true for the uniform shell.

\subsubsection{Theory reminder}

As regards the periodically simply supported finite cylindrical shells, our interest will focus on checking the efficiency of annular ABHs for reducing the vibration transmission from one shell ring to another. To that purpose, a unit ring excitation in the inward radial direction is applied at $z=0.3375 \mathrm{~m}$ (the $z$-axis origin is set at the beginning of the structure) and the radial displacement is computed on a receiver ring, located at $z=13.1625 \mathrm{~m}$ (see Fig.2). The radial displacement transmissibility between the two shell rings is defined as

$$
T_{r s} \equiv 10 \lg \frac{\left\langle w_{r}^{2}\right\rangle}{\left\langle w_{s}^{2}\right\rangle},
$$

where $w_{r}$ and $w_{s}$ denote the radial displacements at the receiver and source rings, and the brackets indicate spatial averaging on the ring circumference. We shall remark that the radial displacement is obviously independent from the circumferential position for the axisymmetrical models and therefore averaging becomes unnecessary for them. However, in Section 4 we will introduce stiffeners in the annular ABHs which will break the axisymmetry and justify the need for averaging in Eq. (6).

The displacements $w_{r}$ and $w_{s}$ can be directly obtained from the solution of the FEM system

$$
\left[\mathbf{K}-\omega^{2} \mathbf{M}\right] \mathbf{q}=\mathbf{f}
$$

with $\mathbf{K}$ standing for the model complex stiffness matrix, $\mathbf{M}$ for the model mass matrix and $\mathbf{f}$ for the external nodal force vector, whose components are zero except for the radial ones at the excitation rings. The vector q contains the nodal displacements from which we can extract the radial component ones $w_{r}$ and $w_{s}$.

On the other hand, periodic boundary conditions have to be imposed in the circumferential direction given that we are only simulating one sector of the cylindrical shell. Suppose that we divide the shell into $N$ sectors (in our case $N=16$ ). Let us denote by $\mathbf{q}_{A}$ the nodal displacement vector of the bottom boundary in the $z$-direction of the simulated sector, and by $\mathbf{q}_{A}^{\prime}$ the nodal displacement vector at the top boundary (see Fig.2). Analogously, let us designate by $\mathbf{q}_{B}$ and $\mathbf{q}_{B}^{\prime}$ the bottom and top displacement vectors at any other sector. It then follows that the displacement vectors of both sectors can be related through

$$
\left(\begin{array}{l}
\mathbf{q}_{A}^{\prime} \\
\mathbf{q}_{B}^{\prime}
\end{array}\right)=\left(\begin{array}{cc}
\cos (k \alpha) & -\sin (k \alpha) \\
\sin (k \alpha) & \cos (k \alpha)
\end{array}\right)\left(\begin{array}{c}
\mathbf{q}_{A} \\
\mathbf{q}_{B}
\end{array}\right)
$$

with $k$ standing now for the wavenumber in the circumferential direction and $\alpha=2 \pi / N$.

\section{Simulations for uniform and $\mathrm{ABH}$ non-stiffened infinite and finite shells with periodic simple supports}

\subsection{Passbands, stopbands and transmissibility for the uniform shells}

To begin with, let us first focus on the results for the infinite uniform shell with periodic supports. The dispersion curves for that shell in the frequency range [0 1000] Hz have been calculated according to the theory in Section 2.1.2 and are presented in Fig.3a. As usual, these curves are computed for an equivalent conservative system for simplicity (see e.g., [17] and references therein), though the mass, stiffness and loss 

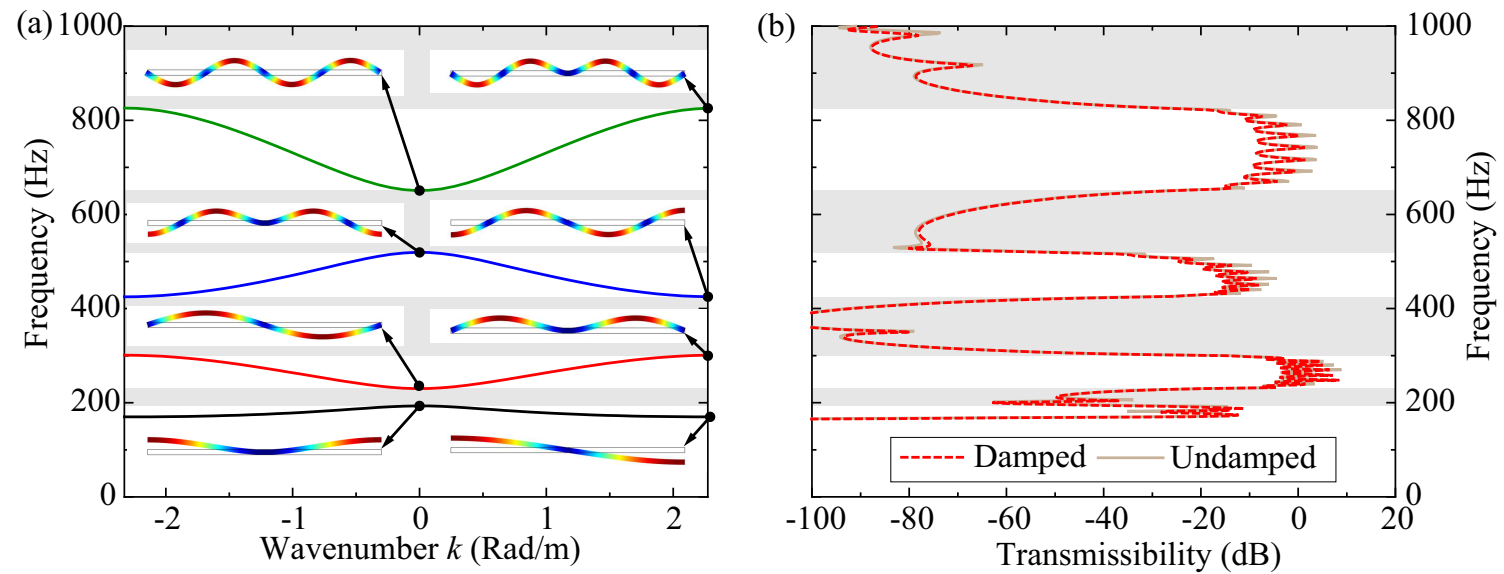

Figure 3: (a) Dispersion curves and mode shapes for the undamped infinite uniform shell. (b) Transmissibility for the finite 10-cell uniform shell, with and without damping layers.

factor of the damping layer are taken into account in the transmissibility plot of Fig.3b. If one compares the stopbands and passbands in the two figures no significant differences can be appreciated.

It is observed in Fig.3a that no dispersion curve appears below the ring frequency of the shell, $\omega_{R}=$ $R^{-1} \sqrt{E / \rho}$, which for the present example turns to be $f_{R}=\omega_{R} / 2 \pi \approx 173 \mathrm{~Hz}$. This is not surprising since in this paper we are focusing on the shell low frequency vibration that is dominated by flexural motion. The latter is the most critical one e.g., in terms of noise radiation [17]. In particular, we consider bending-type waves with zero wavenumber in the circumferential direction. It was shown in [2, 3] that the bounding frequencies of the passbands of symmetric periodic structures correspond to the eigenfrequencies of their unit cell submitted to different types of boundary conditions. In the case of cylindrical shells, the dispersion relation for a mode with zero-th circumferential order and arbitrary boundary conditions is of the type $\omega^{2}=D\left(\rho t_{u}\right)^{-1} k^{4}+w_{R}^{2}, D$ being the shell stiffness (see e.g., Eq. (23) in [55] and also [56]). This clearly imposes that wave motion will take place for frequencies higher than $\omega_{R}$.

On the other hand, it is seen in Fig.3a that there exist four passbands and four stopbands in the range up to $1000 \mathrm{~Hz}$. The vibration shapes at $k=0$ and $k=\pi / L_{u} \approx 2.33 \mathrm{rad} \mathrm{m}^{-1}$ of each passband have been also plotted to demonstrate the flexural nature of the motion. If one next plots the displacement transmissibility between the source and receiver rings in the finite ten-cell uniform shell, it becomes apparent that the minima in the transmissibility function coincide with the stopbands of the infinite structure, while the maxima do so for the passbands, see Fig.3b (Note that the range in the horizontal axis of the transmissibility has been adapted for visualization purposes, values below $f_{R}$ being negligible). The differences between maxima and minima always exceed $60 \mathrm{~dB}$, which is remarkable. Another notorious aspect from the figure is the almost negligible influence the damping layer has on the vibration transmission. If one compares the red curve (undamped uniform cylinder) with the gray one (damped uniform cylinder), the viscoelastic layer is only capable of reducing a few $\mathrm{dBs}$ at the maxima of the transmissibility function.

While the periodicity of the structure results in a very efficient way to prevent the transmission of vibrations at stopbands, see Fig.3b and the displacement shape of the finite shell at the center of the third stopband in Fig.4a, the opposite occurs at passbands. BF waves can propagate with very little attenuation, even if damping layers are attached to the shell to try to mitigate transmission (see again Fig.3b and also Fig.4b, where we plot the displacement shape of the cylinder at the center of the fourth passband). It will be shown in next section how such propagative BF waves can be strongly reduced by means of annular ABHs.

\subsection{Passbands, stopbands and transmissibility for the $A B H$ shells}

Let us next consider the case of a periodically simply supported cylindrical shell with annular ABHs embedded in it, as depicted in the unit cell scheme of Fig.1b. As for the uniform case, we start presenting the dispersion curve for this model in Fig.5a as well as some modal shapes for $k=\left\{0, \pi / L_{u}\right\}$. Two interesting 

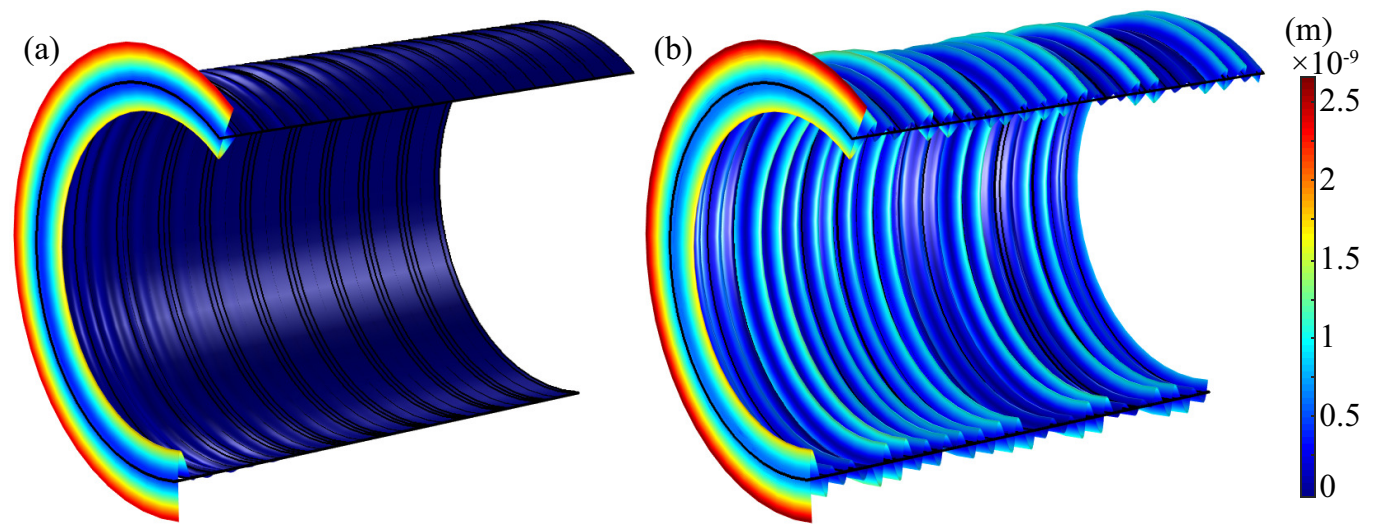

Figure 4: Displacement shape for the uniform shell at (a) $585 \mathrm{~Hz}$ (center of the third stop band), (b) $738 \mathrm{~Hz}$ (center of the fourth pass band)

features stand out. On the one hand, the stiffness of the shell diminishes because of the material removed for tailoring the ABHs. This lowers the shell eigenfrequencies. The consequence is a larger number of stopbands and passbands in the $[0$ 1000] Hz frequency range, as compared to that of the uniform shell in Fig.3a. On the other hand, it is worth noting that beyond the ring frequency of $173 \mathrm{~Hz}$, the first three stop bands get extremely close one to another. This implies that wave propagation is almost forbidden for frequencies below $420 \mathrm{~Hz}$, which is very advantageous for the control of vibrations at low frequencies.

Beyond $420 \mathrm{~Hz}$, however, several passbands exist which may result in considerable shell vibration levels. This is clearly appreciated in Fig.5b, where the transmissibility between the source and receiver rings has been plotted for the finite $\mathrm{ABH}$ shell of Fig.2. If one considers the shell with embedded annular acoustic black holes but without damping (gray line in Fig.5b), the ABH effect is hardly noticed and the situation closely resembles that encountered for the uniform shell in Fig.3b, with strong peak values at the frequency passbands. This is not surprising since from its very first proposal, ABHs were known to totally fail if energy was not dissipated at their centers $[19,20]$. The inclusion of the viscoelastic damping layer drastically changes the situation (red line in Fig.5b). The energy concentrated at the ABH center gets mostly dissipated and, as seen in the figure, the transmissibility levels strongly diminish.

One should bear in mind that the performance of $\mathrm{ABH}$ shells at low and high frequencies are quite distinct. The former is not related to the $\mathrm{ABH}$ effect but to variations in mass and stiffness on the holding
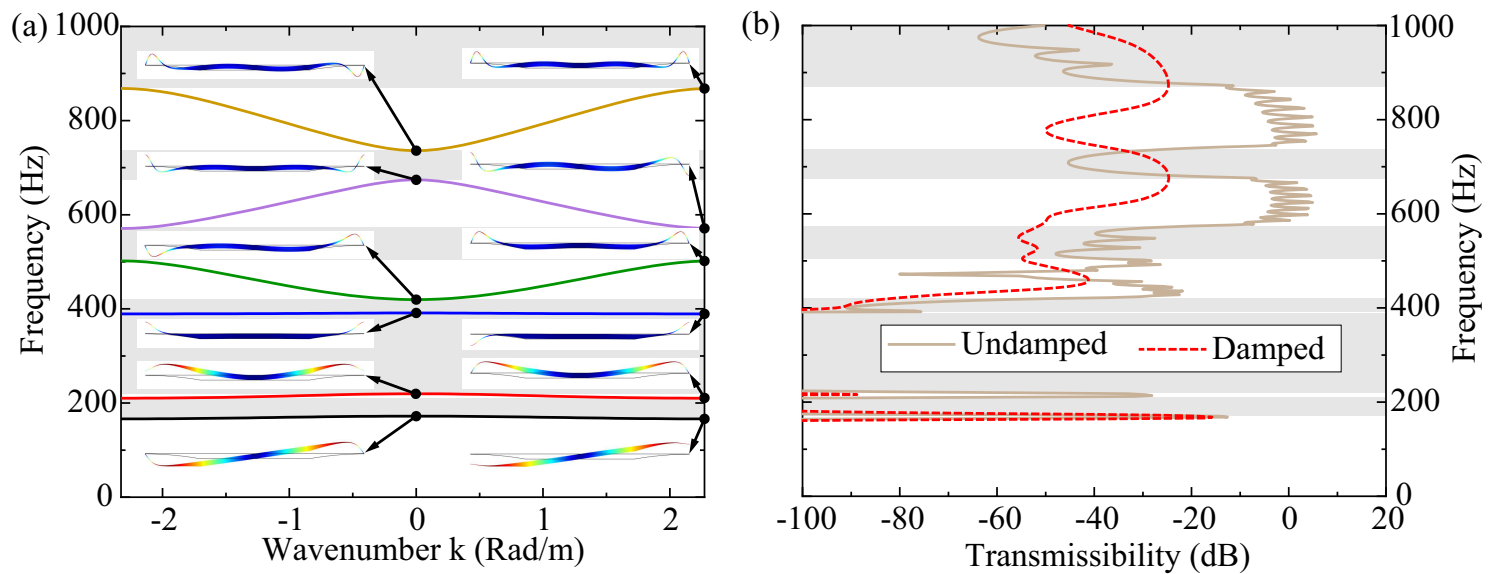

Figure 5: (a) Dispersion curve and mode shapes for the infinite undamped ABH shell. (b) Transmissibility for the finite 10-cell ABH shell, with and without damping layers. All the parameters are referred to Table 1. 

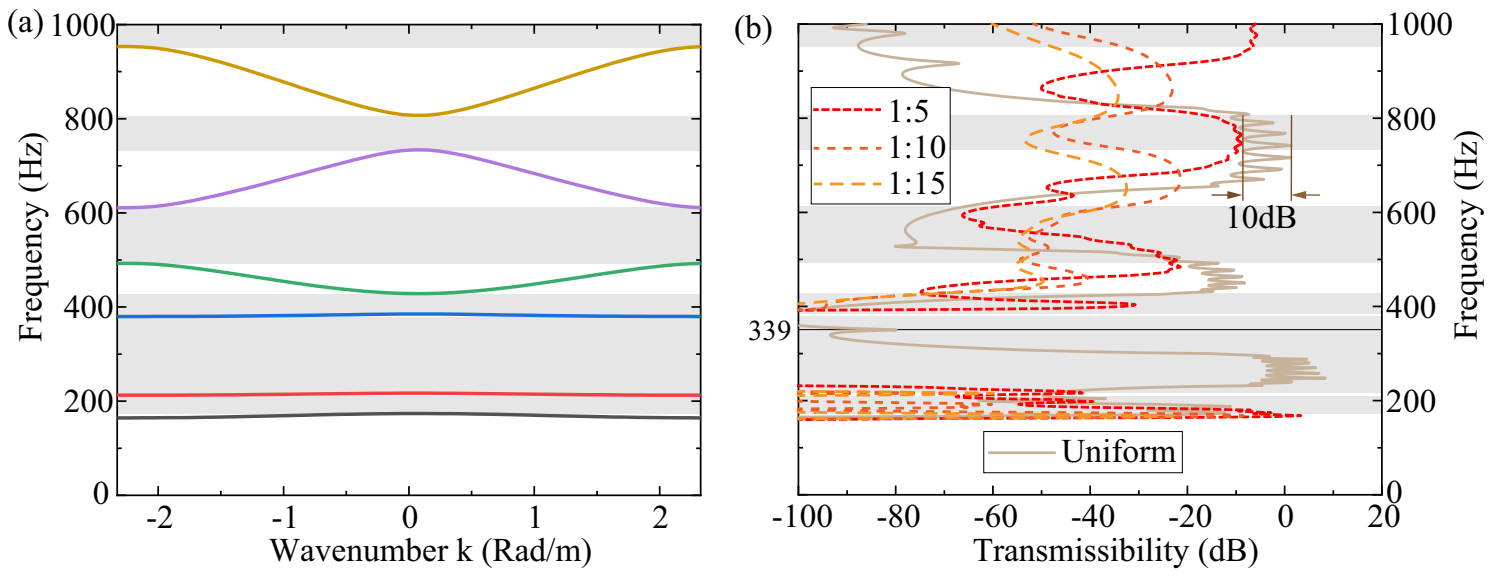

Figure 6: (a) Dispersion curve for the infinite damped ABH shell. (b) Transmissibility between the receiver and source rings in the finite shell model of Fig.2 for different residual thicknesses.

structure due to $\mathrm{ABH}$ indentations. The effects are therefore very case dependent and in the present example have resulted in the nearly merging of the three first stopbands. Yet, at mid to high frequencies (those depending on the $\mathrm{ABH}$ dimensions and profile), is where the $\mathrm{ABH}$ effect starts functioning. In fact,
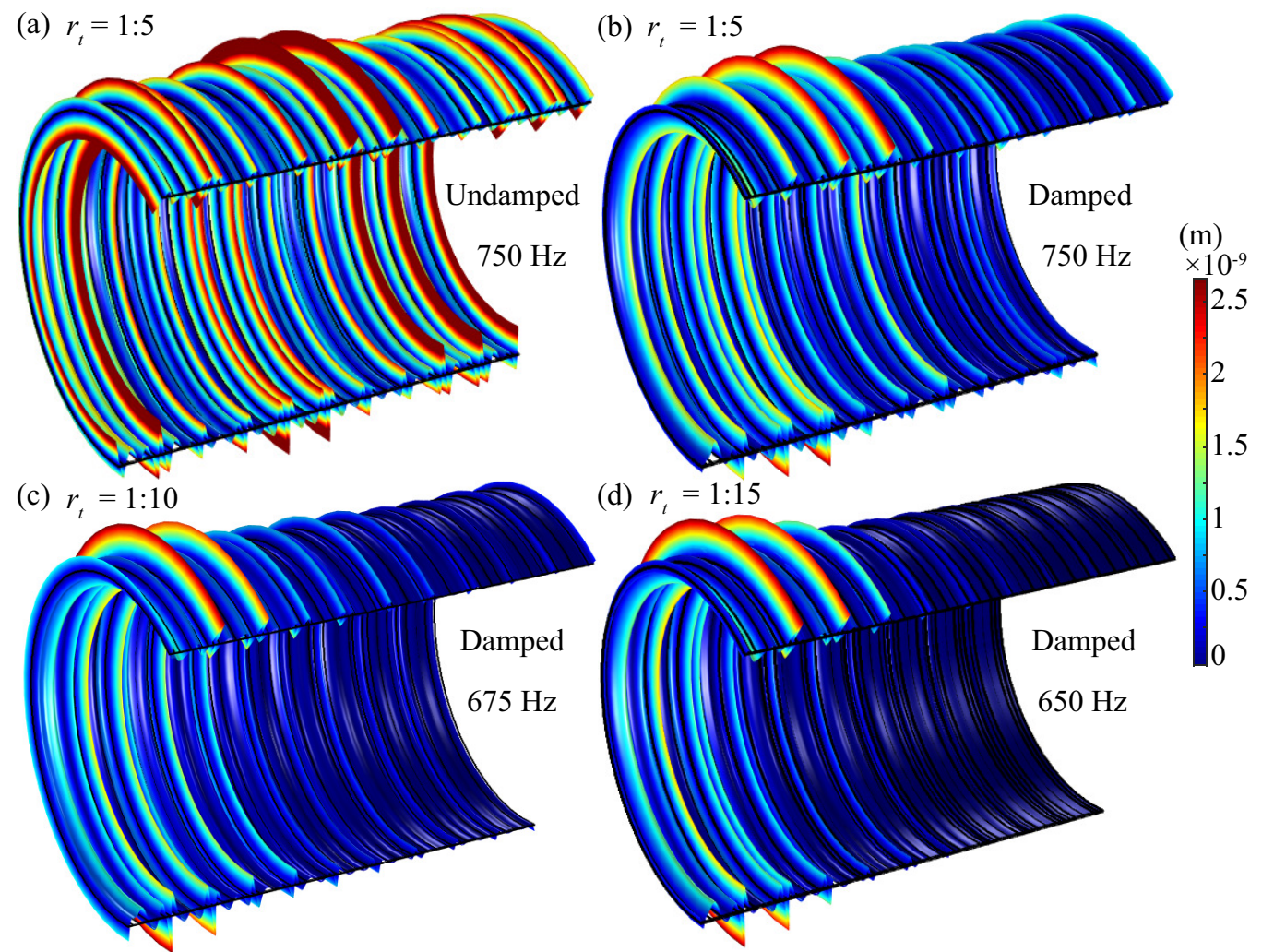

Figure 7: Vibration shapes for ABH shells of different residual thickness ratio at the central frequency of their sixth passband, (a) $r_{t}=1: 5$ without damping layer, (b) $r_{t}=1: 5$ with damping layer, (c) $r_{t}=1: 10$ with damping layer, and (d) $r_{t}=1: 15$ with damping layer. 


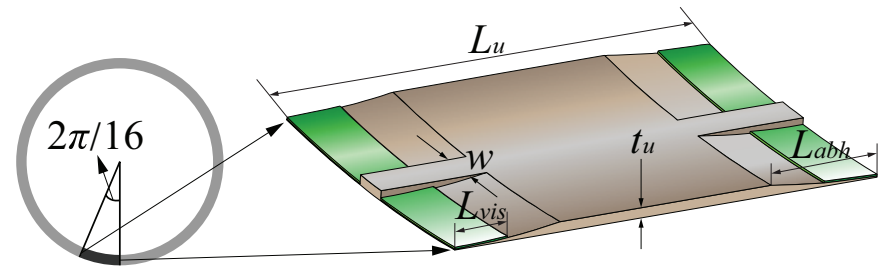

Figure 8: Sketch of a unit cell segment with annular ABH plus stiffener.

that occurs beyond the so-called diameter cut-on frequency $f_{\mathrm{abh}}=\frac{\pi t_{u}}{4 L_{\mathrm{abh}}} \sqrt{\frac{E}{3 \rho}}$ (see e.g., [28, 30, 35, 37]). Essentially, the latter establishes that the wavelength of a wave entering the $\mathrm{ABH}$ needs to be smaller than the $\mathrm{ABH}$ diameter (in this case the length $L_{\mathrm{abh}}$ ) to perceive its effects. However, the $\mathrm{ABH}$ does not become fully operative until the so called smoothness cut-on frequency, $f_{\varepsilon}=\frac{\varepsilon}{2 \pi} \sqrt{\frac{E}{3 \rho}}$, is surpassed (see e.g., $[19,28,38,41]) . f_{\varepsilon}$ is related to the $\mathrm{ABH}$ profile and guarantees that an incident wave on the ABH finds a smooth impedance matching so that it not gets reflected back. In other words, for a fixed ABH order $m$, if the smoothness parameter $\varepsilon$ is too large an incoming wave would almost experience impinging on a straight wall. But, if $\varepsilon$ is small enough, the wave will smoothly enter the $\mathrm{ABH}$. In practice, the $\mathrm{ABH}$ effect becomes fully effective for frequencies $f \gtrsim 3 f_{\varepsilon}$ (see e.g., [30]).

Given that the ABH thickness gets very thin close to its center, the mass and stiffness of the damping layer may play a significant role there. Consequently, in Fig.6a we start presenting the dispersion curves for the $\mathrm{ABH}$ taking the latter into account. Those are to be compared with the ones of the undamped ABH in Fig.5a. One can appreciate variations in the widths and locations of some of the stopbands and passbands, especially at higher frequencies, though the number of bands and their behavior remain the same in the analyzed frequency range. Besides, the influence of a critical parameter that affects the efficiency of ABHs, namely the truncation thickness, $t_{0}$, is analyzed in Fig.6b. Its influence has been reported in several works, see e.g. [21, 26, 30, 57] among others, and it is constructive to consider it in the present situation. To that purpose, let us introduce the thickness ratio, $r_{t}=t_{0} / t_{u}$, and see what happens when we modify it. We note that the transmissibilities already shown in Fig.5b correspond to an annular ABH with thickness ratio $r_{t}=1: 10$. Those for larger and smaller ratios, namely $r_{t}=1: 5$ and $r_{t}=1: 15$, are presented in Fig.6b together with the uniform and $r_{t}=1: 10$ cases, for comparison. The latter has a three times smoothness frequency value $3 f_{\varepsilon}=339 \mathrm{~Hz}$ (plotted in the figure), which gets slightly modified to $3 f_{\varepsilon}=301 \mathrm{~Hz}$ for the $r_{t}=1: 5$ case and to $3 f_{\varepsilon}=351 \mathrm{~Hz}$ for the $r_{t}=1: 15$ one. It can be observed in the figure how the peak locations move to lower frequencies as the thickness ratio decreases because the shell stiffness diminishes. More remarkably, it becomes apparent from the figure that very strong vibration reductions can be achieved with the ABHs. Even in the weakest case of the $r_{t}=1: 5$ ratio, the transmissibility peaks lessen $\sim 10 \mathrm{~dB}$ compared to those of the uniform shell.

To better illustrate the residual thickness influence on the annular ABH performance, in Fig.7 we have represented the shell vibration shapes for different thickness ratios, at the central frequency of their sixth passband. Figs.7a and 7b show those corresponding to the $r_{t}=1: 5$ case at $750 \mathrm{~Hz}$ and reveal the critical importance of including a viscous damping layer to achieve the $\mathrm{ABH}$ effect. The vibration reduction improves as we move away from the excitation ring as one could expect. In fact, it becomes almost negligible at the farthest cells as the thickness ratio diminishes, as observed in Fig.7c for $r_{t}=1: 10$ at $675 \mathrm{~Hz}$ and in Fig.7d, for $r_{t}=1: 15$ at $650 \mathrm{~Hz}$.

\section{Simulations for stiffened ABH finite shells with periodic simple supports}

\subsection{Stiffened annular ABH shells}

In the preceding section, it has been shown that annular ABHs constitute a very effective way of suppressing BF waves in periodically supported cylindrical shells. Notwithstanding, embedding ABHs on the shell weakens its stiffness and this can be problematic from a structural point of view. To partially mitigate 
that problem, we will next explore the effects of including some stiffeners into the ABHs. A sketch of the proposed stiffened cylindrical ABH unit cell was presented in Fig.1c and geometrical details for one of its segments are depicted in Fig.8. As observed, the stiffener has the length of the $\mathrm{ABH}, L_{\mathrm{abh}}$, a width $w$ and the shell thickness $t_{u}$. Each annular ABH contains several stiffeners that continuously connect the periodically arranged cells.

The influence of the stiffeners is basically characterized by two parameters, namely their width and number in a unit cell. As for the former, too wide stiffeners may damage the ABH effect, limiting the energy dissipation area at the $\mathrm{ABH}$ center and thus reducing its performance. Too narrow stiffeners, on the other side, may simply not provide enough structural rigidity. Besides, the choice of the stiffener width must be made in combination with the number of them to be placed in the ABH. The proper width and number of stiffeners for annular ABHs will be examined in forthcoming sections.

\subsubsection{Effects of modifying the stiffener width}

We start considering the 10 unit cell model of Fig.2 and check the consequences of changing the width of a total of sixteen stiffeners inserted on each annular ABH. Widths of $w=4 t_{u}$ and $w=16 t_{u}$ are contemplated. The resulting transmissibilities are plotted in Fig.9a and compared to that of the annular ABH without
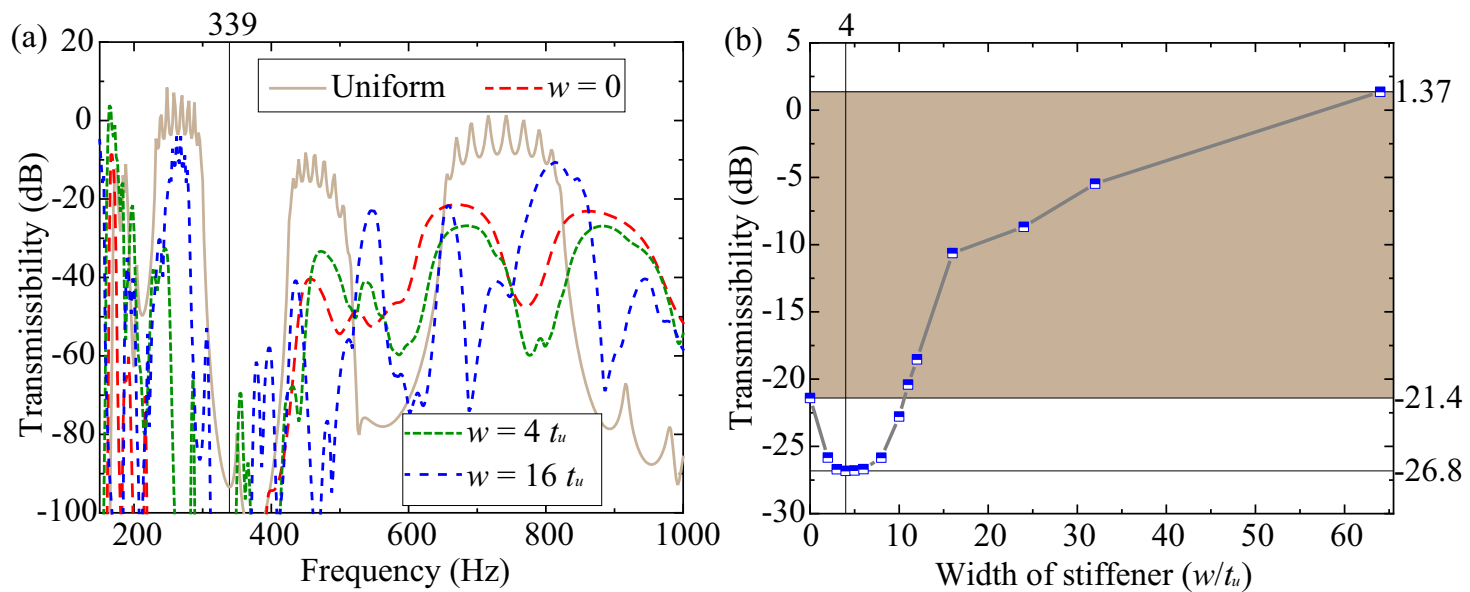

Figure 9: (a) Transmissibility for the different width of stiffeners. (b) Peak values of transmission beyond the smooth cut-on frequencies in term of various stiffener width, with the bare ABH structure corresponding to $-21.4 \mathrm{~dB}$ and the uniform structure to $1.37 \mathrm{~dB}$
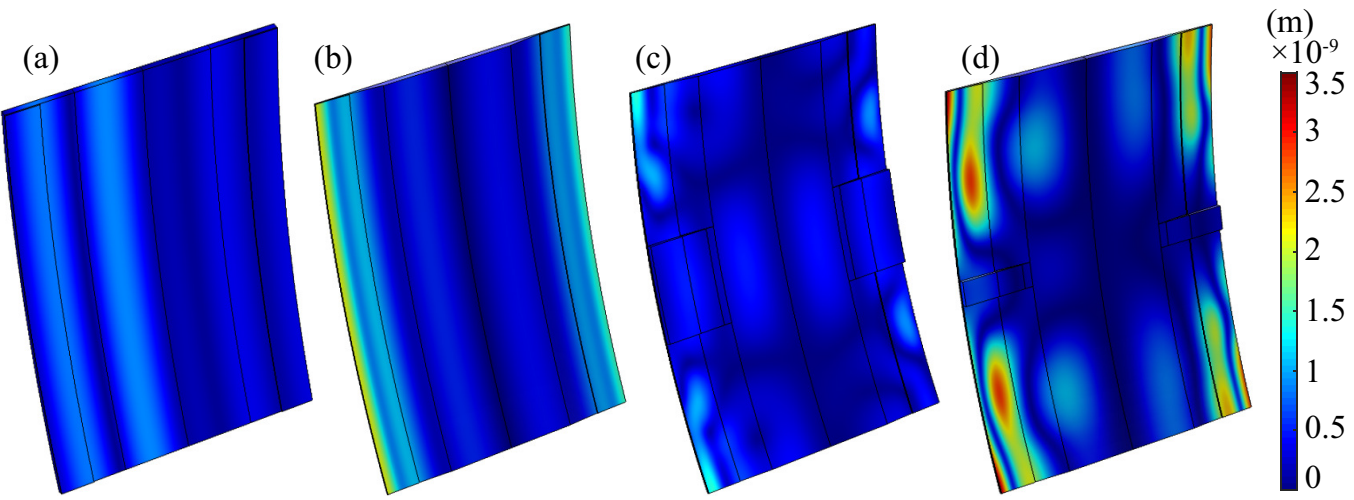

Figure 10: Displacement shape of $1 / 16$ portion of the second cell in the periodically supported finite shell at the frequencies of the global peak values of the transmissibility. (a) Uniform shell at $742 \mathrm{~Hz}$, (b) bare ABH at $672 \mathrm{~Hz}$, (c) stiffened ABH with $w=16 t_{u}$ at $814 \mathrm{~Hz}$ and (d) stiffened $\mathrm{ABH}$ at $675 \mathrm{~Hz}$ with $w=4 t_{u}$. 

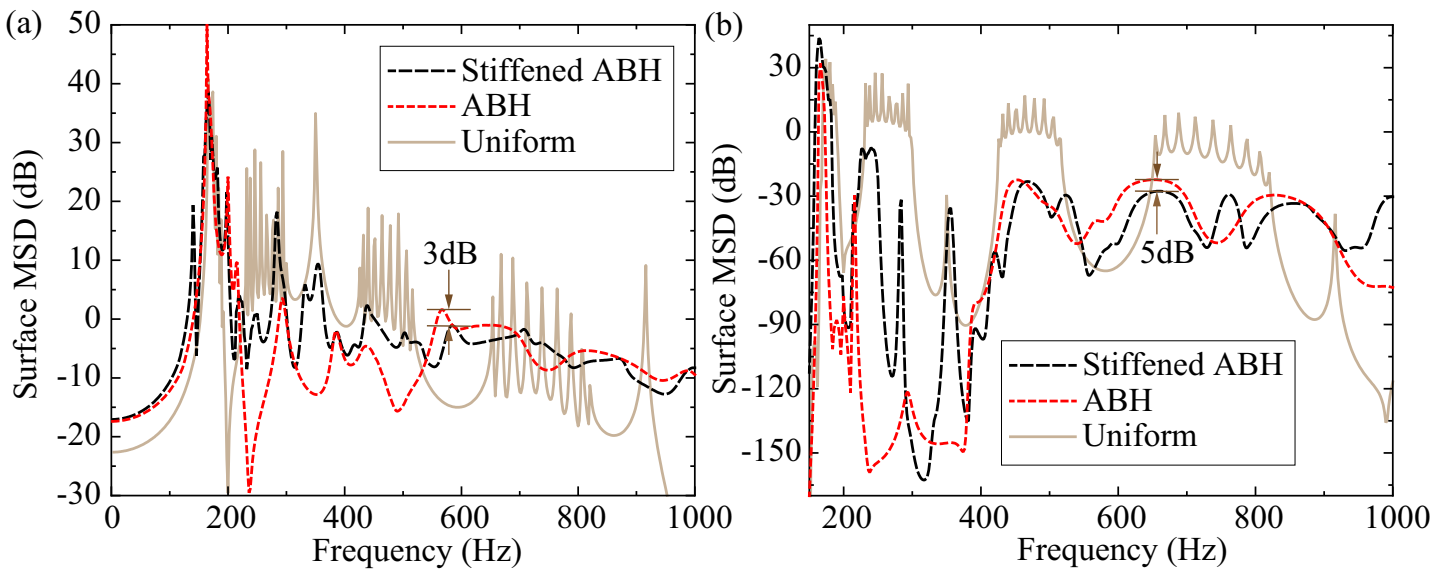

Figure 11: Mean square displacement (MSD)for (a) the second cell and (b) the last cell (reference displacement value $\left.w_{\text {ref }}=1 \times 10^{-9} \mathrm{~m}\right)$.

stiffeners $(w=0)$ and to that of the uniform shell as well.

Two remarkable facts can be appreciated in Fig.9a. The first one is that stiffeners modify the stopband and passband frequency distribution in such a way that the wide non-propagation region of the non-stiffened $\mathrm{ABH}$ comprising from 173 to $420 \mathrm{~Hz}$ gets split, and the passband between 200 and $300 \mathrm{~Hz}$ of the original uniform shell is recovered. The amplitude levels of the transmissibility for the stiffened ABH shells at that passband are yet much smaller than those of the uniform shell. As expected, this is especially the case for $w=4 t_{u}$ but it also happens, to a lower degree, for $w=16 t_{u}$.

The second one concerns the behavior of the stiffened ABHs shells beyond three times the smoothness frequency $3 f_{\varepsilon}=339 \mathrm{~Hz}$. Surprisingly, the $w=4 t_{u}$ stiffened ABH performs better than the bare one, while one would expect the opposite behavior because of the potential vibration transmission through the stiffeners. In fact, this is what one encounters for the $w=16 t_{u}$ case. It is therefore interesting to analyze in more detail the unexpected behavior for $w=4 t_{u}$ and determine whether and why the inclusion of some stiffeners could benefit vibration reduction, without seriously deteriorating the shell rigidity. To that end, Fig.9b illustrates the global peak value of the transmissibility beyond $3 f_{\varepsilon}$ for different values of the width to thickness ratio, $r_{w}$, that being defined as $r_{w}=w / t_{u}$. The upper and lower limits of the brown shaded strip in the figure respectively correspond to the peak values of the periodically supported uniform and bare $\mathrm{ABH}$ shells. One would then predict the maximum values of the stiffened ABHs to lie inside the strip but, as observed, this is not occurring for $r_{w}<10$. It looks like the inclusion of some stiffeners with appropriate width can be actually beneficial to suppress vibrations, the optimal width to thickness ratio value for that enhancement being $r_{w}=4$.

To better understand the underlying mechanism of the above unforeseen behavior, one should bear in mind that the functioning of the $\mathrm{ABH}$ effect relies on the dissipation of the energy concentrated at its center, by means of the viscoelastic layer. Accordingly, in Fig.10 we have examined the displacement shapes of the second cell of the structure at frequencies corresponding to its global maximum transmissibility. The cases of the uniform, $\mathrm{ABH}$ and stiffened $w=16 t_{u}$ and $w=4 t_{u} \mathrm{ABH}$ shells, are shown in the figure. Only 1/16 portion of the second cell is depicted thanks to symmetry and for a better illustration of the results. For the uniform shell, see Fig.10a, the displacement field has circumferential symmetry, as expected from the ring excitation distribution. The vibration becomes slightly higher at the region closer to the source (first unit cell placed to the left, but not shown in the figure) and the damping exhibits negligible effect, as already commented before. In the case of the bare ABH of Fig.10b, the circumferential symmetry is still preserved, but now the vibration mostly concentrates in the $\mathrm{ABH}$ center where it becomes dissipated preventing $\mathrm{BF}$ wave transmission. As for the stiffened ABH shells, we distinguish between the oversized one $\left(w=16 t_{u}\right)$ in Fig.10c, and the optimal one $\left(w=4 t_{u}\right)$ of Fig.10d. For the former, the stiffeners deteriorate the $\mathrm{ABH}$ performance and the results lie between those of the bare $\mathrm{ABH}$ shell and the uniform shell, as reported in 

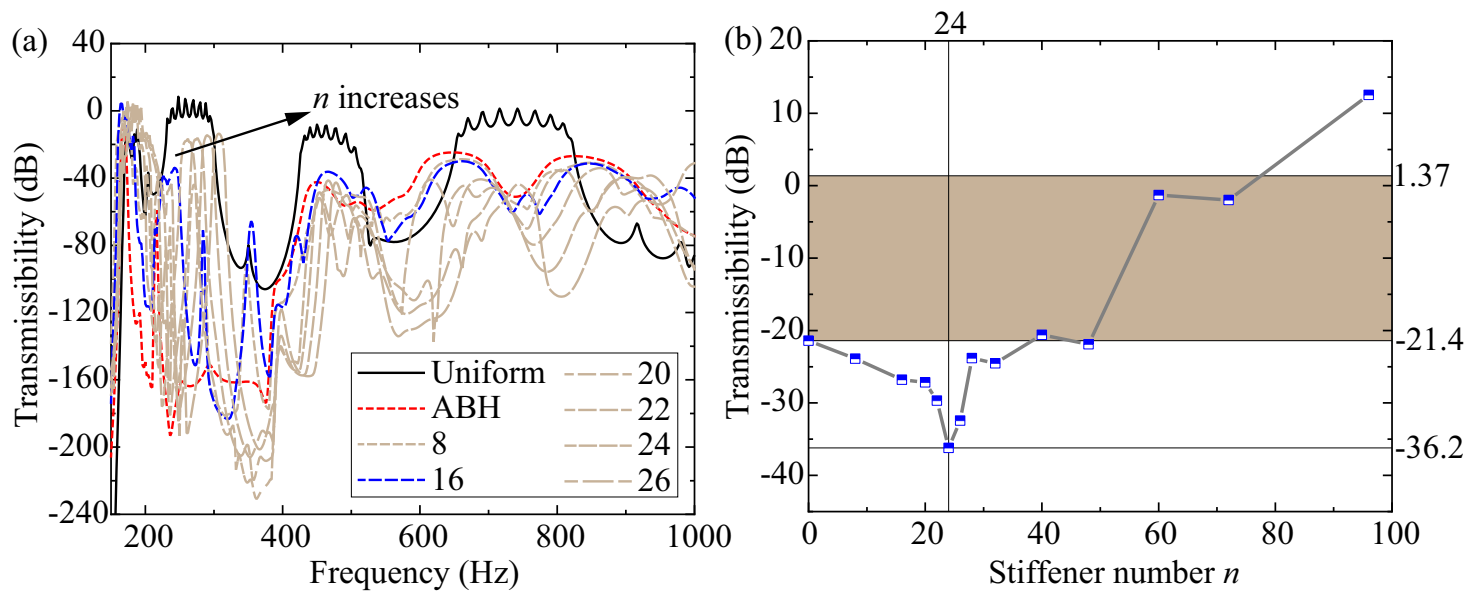

Figure 12: Transmissibility of the ABH shells when modifying the number of stiffeners.
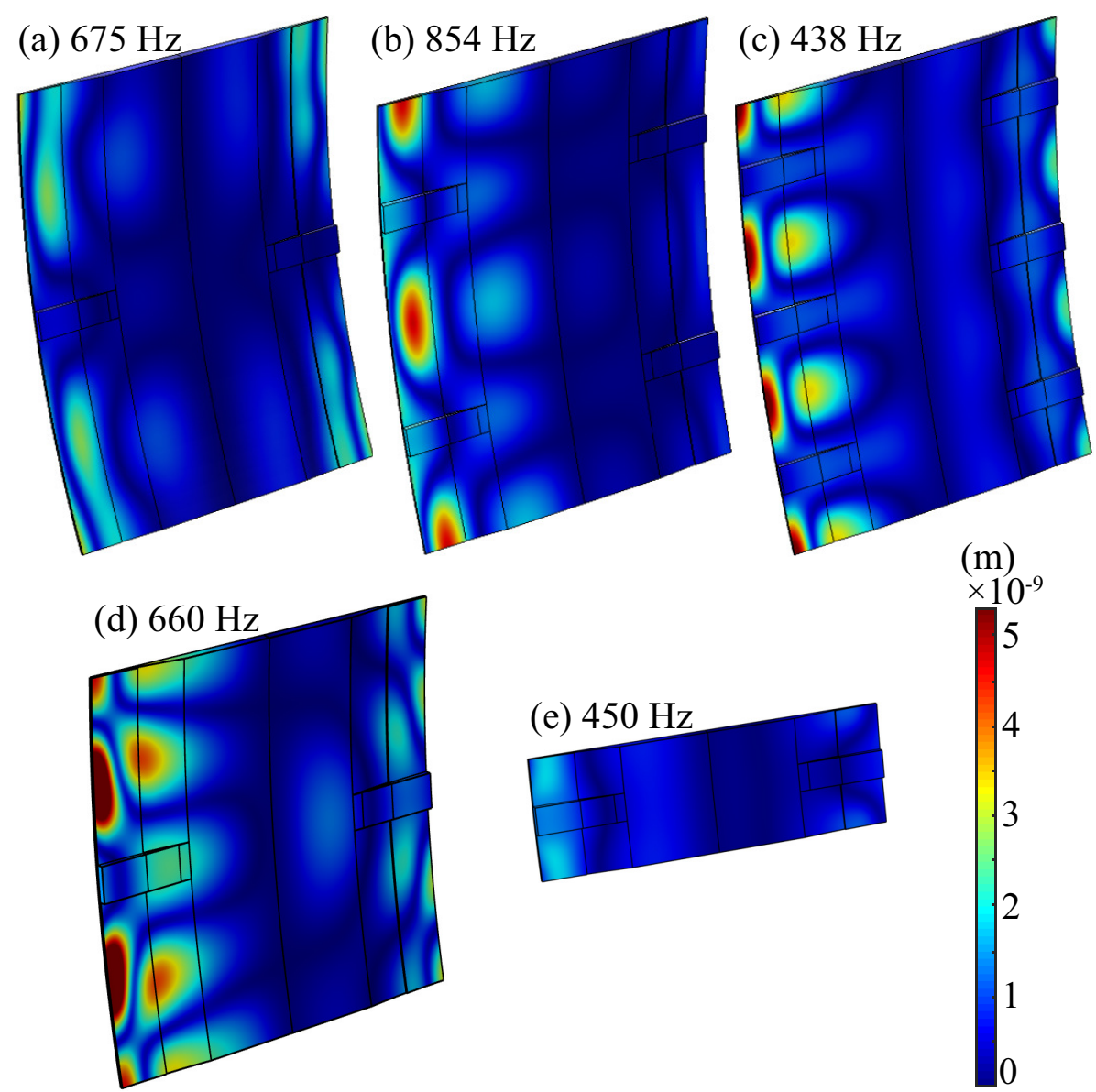

Figure 13: Displacement distribution for the rigid ABH shells with (a) 1 , (b) 2 and (c) 3 stiffeners for $1 / 16$ section, (d) 1 stiffener for $1 / 24$ section and (e) 1 stiffener for $1 / 60$ section of the second cell.

Figs.9a and 9b. Nonetheless, in the optimal case, $w=4 t_{u}$, the stiffeners seem to help focusing the energy on the ABH central dissipation area, which enhances the efficiency of the non-stiffened annular ABH. 


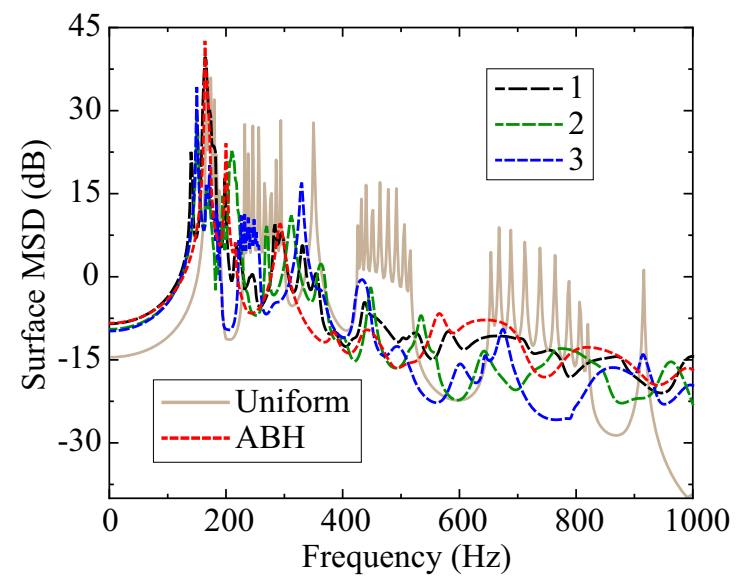

Figure 14: Overall surface mean square displacement (MSD) for the 10-cell shell for different number of stiffeners (reference displacement value $w_{\text {ref }}=1 \times 10^{-9} \mathrm{~m}$ ).

To conclude this subsection and to better appreciate the efficacy of the annular ABHs, it is worth having a look at the cells surface mean square displacement (MSD). In Figs.11a and 11b we respectively present the MSD dependence on frequency of the second and last unit cells, for the uniform, ABH and optimal stiffened ABH shells. Beyond $3 f_{\varepsilon}$, the stiffened $\mathrm{ABH}$ shell is able to reduce the peak values of the bare $\mathrm{ABH}$ from 3 to $5 \mathrm{~dB}$. At low frequencies however, say below $400 \mathrm{~Hz}$, the latter performs better because of the splitting of the first frequency stopband, as mentioned before. Both, the stiffened and non-stiffened ABH shells substantially diminish the MSD values found for the uniform shell.

\subsubsection{Influence of the number of stiffeners}

Once analyzed the influence of the stiffener width on the ABH performance, let us address the effects of varying their quantity. Fig.12a plots the transmissibility between the receiver and source rings when changing the number $n$ of stiffeners per ABH. A stiffener width of $w=4 t_{u}$ has been considered in all simulations. Given that many values of $n$ have been examined, only the curves for the uniform, bare ABH, and $\mathrm{ABH}$ with 16 stiffeners have been highlighted in different colors. The curves for the remaining values of $n$ have uniform color and the tendency of their transmissibility dependence on frequency is indicated with an arrow in the figure. As $n$ augments, the transmissibility slightly worsens at low frequencies as the peaks tend to increase and move to higher frequencies because the rigidity intensifies. For higher frequencies, however, the influence of the number of stiffeners seems to be rather complex, as observed in Fig.12b, which is the analogous of Fig.9b. The figure indicates an optimum value of $n=24$ stiffeners, yet one should be

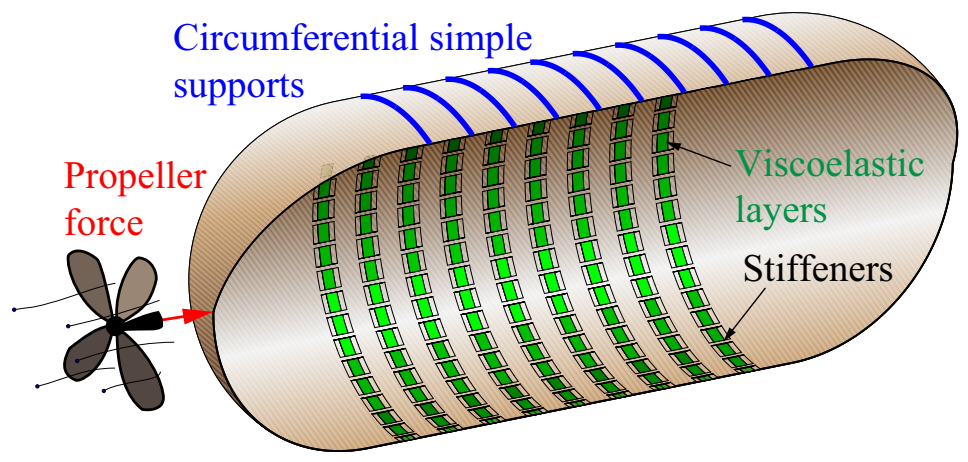

Figure 15: A closed periodically supported cylindrical shell with stiffened annular ABHs that could be viewed as a very schematic model of the hull of a submarine vehicle. 

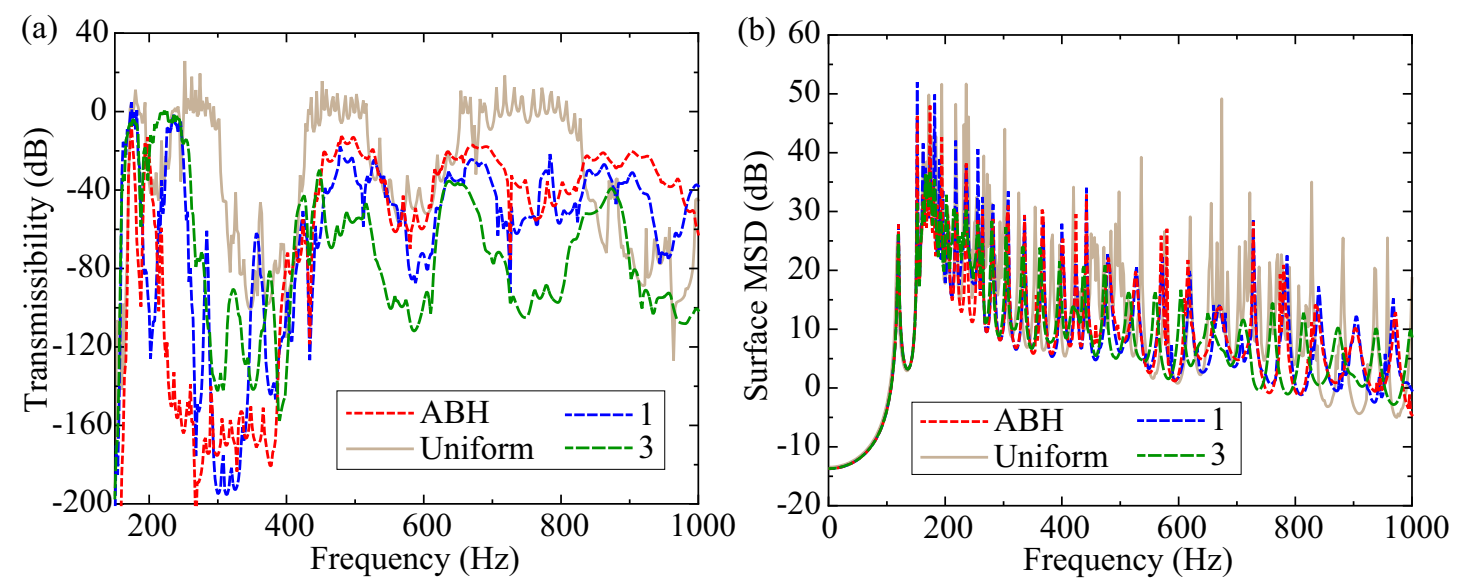

Figure 16: (a) Transmissibility and (b) Mean square displacement (MSD) for the closed cylindrical shell of Fig.12. Efficiency of the bare and stiffened and annular ABHs. The reference value is chosen as $w_{\text {ref }}=1 \times 10^{-9} \mathrm{~m}$.

cautious with the outputs of that figure. The $n=24$ dip is very sharp and for $n=96$ we get much worse results than for the uniform shell.

It is therefore illustrative to inspect the displacement shapes for different configurations, as we previously did in Fig.10 for varying stiffener widths. Figs.13 now depicts 1/16 portion of the second shell for 1 (Fig.13a), 2 (Fig.13b) and 3 (Fig.13c) stiffeners. Those respectively correspond to 16, 32 and 48 stiffeners for each complete annular ABH. Besides, 1/24 portion (Fig.13d) and 1/60 portion (Fig.13e) of the second shell with 1 stiffener are also presented, respectively accounting for 24 and 60 stiffeners on a complete annular $\mathrm{ABH}$. Clearly, the $1 / 24$ portion case is the one that helps concentrating more energy at the ABH centers to dissipate energy. An excessive number of stiffeners, however, undermines energy concentration in the $\mathrm{ABH}$ areas (see Fig.13e). Finally, we have also plotted the overall surface MSD for the last cell in Fig.14 to check the efficacy in reducing BF wave propagation. It can be realized that the MSDs of the stiffened ABH shells are higher than those of the bare ABH shell in the $[200500] \mathrm{Hz}$ frequency interval. The situation reverses within $[5001000] \mathrm{Hz}$ for the 48 stiffener configuration (i.e. 3 stiffeners in the 1/16 portion) which performs better in that range.

\subsection{A closed periodically supported cylindrical shell with annular ABHs}

In the precedent examples, we have considered the case of a periodically supported cylindrical shell of infinite length as well as a finite one with open ends. In practical applications, those models may be devised as simple approximations for industrial long pipelines, air-conditioning ducts, etc. However, cylindrical structures with closed ends are also very common. One may think, for instance, of airplane fuselages, hulls of submarine vehicles, or gas and fluid tanks, to mention a few. To check to what extent annular ABHs may prove also useful in those situations, we have constructed a new model by closing the ten-cell finite model with two spherical caps (see Fig.15). The caps are $5 \mathrm{~m}$ in radius and have replaced the first and last unit cells of the finite open cylindrical shell. The cases of a uniform closed shell, a shell with nine annular $\mathrm{ABH}$ s and a shell with nine annular stiffened $\mathrm{ABHs}$ have been investigated. As for the latter, a width to thickness ratio of $r_{w}=4$ has been assumed for ABHs containing 16 and 48 stiffeners $(1$ and 3 stiffeners respectively for $1 / 16$ of the structure). A point excitation has been applied at the center of the first cap, in the longitudinal direction, which might represent e.g., the main propeller shaft force component in the hull of a submarine vehicle.

Transmissibility (between the excitation point and the opposite end point) results are shown in Fig.16a. Despite of the curves being rougher than those encountered for the open ten cells model (see Fig.9a and Fig.12a), the presence of stopbands and passbands can be clearly appreciated in the figure. Their frequency ranges match with those already found for the open finite shell model. Also, the performance of the annular 

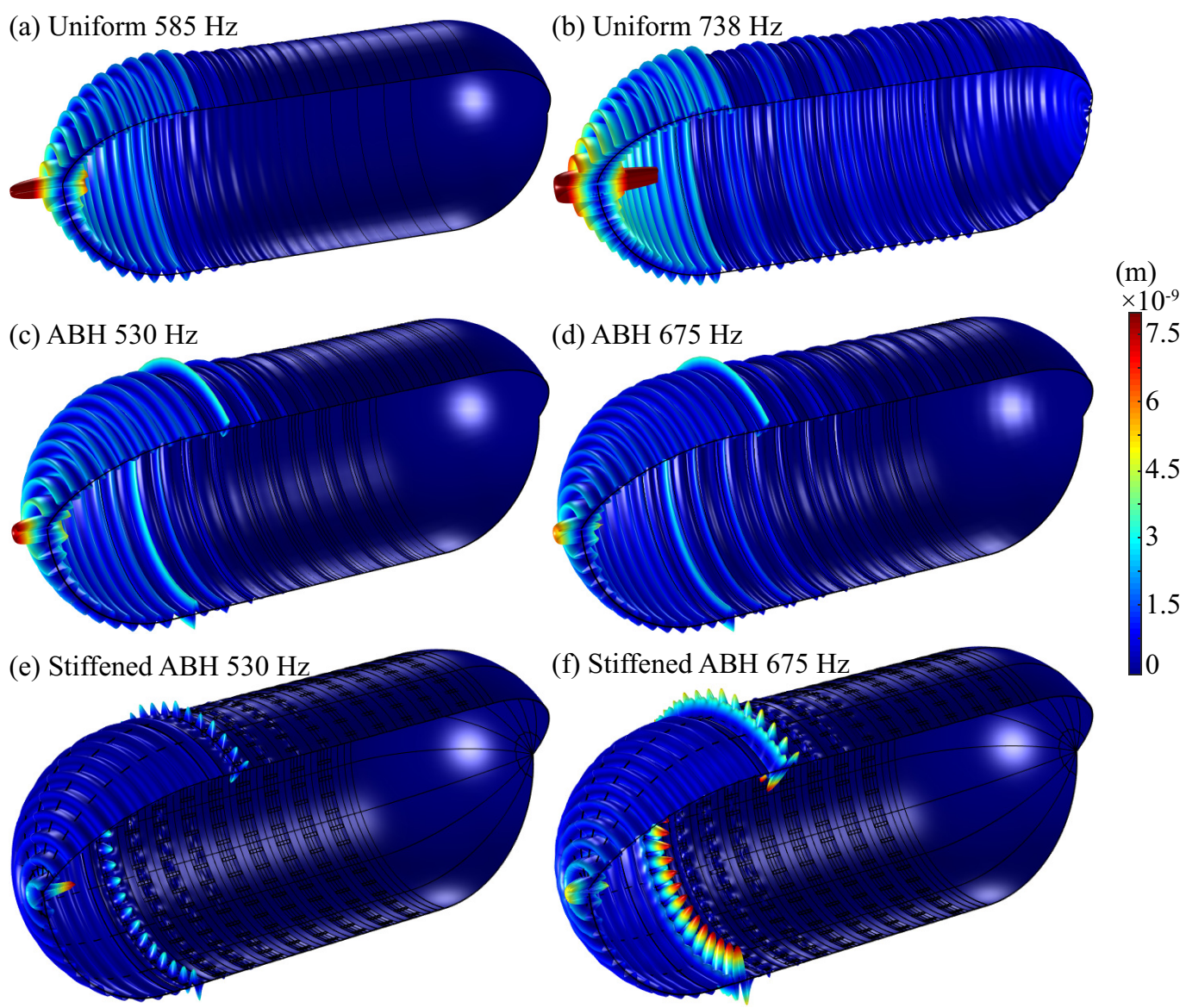

Figure 17: Displacement vibration shapes for (a)-(b) the uniform shells at the third stopband and fourth passband central frequency, (c)-(d) ABH shells, (e)-(f) stiffened ABH shells. Subfigures (c) and (e) correspond to the fifth stopband of the bare $\mathrm{ABH}$ structure, while (d) and (f) to its sixth passband. The stopbands and passbands have been selected for better illustrative purposes.

ABHs for the closed shell is in close agreement with that found for the open one. The embedding of the ABHs noticeably reduces the transmissibility values and, in particular, setting 48 stiffeners in them enhances their behavior even more. As for the overall surface MSD, this has been plotted in Fig.16b showing a strong reduction of the MSD peak values at all frequencies.

Finally, and similarly to what was done in Fig.4 and Fig.7, we present the displacement vibration shapes of the uniform, ABH and 48-stiffened ABH shells at the central frequencies of some stopband and passbands, in Fig.17. The first column in the figure corresponds to stopbands and the second one to passbands. As one could expect almost no vibration is transmitted for the stopbands (Figs.17a, 17c and 17e). The opposite logically occurs at the passbands and, as clearly observed in Fig.17d and Fig.17f, the bare and stiffened ABHs constitute a very effective way to reduce vibration transmission. Another possibility for further reduction, not contemplated in this work, would be to set a further ring-shaped ABH encircling the structural excitation point, following the key idea in [37].

\section{Conclusions}

This paper proposes the design of annular acoustic black holes (ABHs) to reduce the propagation of bending Bloch-Floquet (BF) waves in periodically simply supported cylindrical shells. Wave finite element models (FEM) have been used to predict the location of frequency stopbands and passbands in uniform and 
ABH infinite shells. It has been shown how the inclusion of annular ABHs can strongly affect those bands. Most importantly though, it has been checked by means of the FEM model of a periodically supported shell consisting of ten cells that the $\mathrm{ABHs}$ drastically reduce the vibration transmissibility. Unfortunately, this remarkable output has a well-known counterpart. The ABH indentations weaken the shell rigidity and therefore deteriorate its structural performance.

To partially remedy the rigidity problem, we have analyzed the effects of inserting stiffeners in the annular ABHs. Two key parameters have been considered: the stiffeners' width and their number. Quite surprisingly, it has been found that for some proper combinations of the latter, the stiffeners not only do not worsen the $\mathrm{ABH}$ effect but they actually enhance it. The reason for that is the stiffeners help concentrating the energy at the center of the ABHs, where it gets dissipated by the viscoelastic damping layer. This topic deserves further exploration and in the future optimization techniques could be used to find an optimum distribution and size of stiffeners, which guarantees a good balance between vibration suppression and structural requirements.

Finally, the case of a cylindrical structure with closed ends and periodic supports has been considered as a first step towards more realistic geometries found in the aeroanautical and naval sectors. Similar results to those of the infinite and open end finite shells have been reported, showing the potential to apply annular $\mathrm{ABHs}$ to realistic structures.

\section{Acknowledgments}

This work has been completed while the first author was performing a two-year PhD stay at La Salle, Universitat Ramon Llull, funded by the National Natural Science Foundation of China under Grant (51875061) and the China Scholarship Council (CSC No.201806050075). The authors gratefully acknowledge this support as well as the in-kind assistance from La Salle, Universitat Ramon Llull, and the Chongquing University to make that collaboration possible. The second author would also like to thank l'Obra Social de la Caixa and the Universitat Ramon Llull for their support under grant 2018-URL-IR2nQ-031.

\section{References}

[1] D. Mead, Free wave propagation in periodically supported, infinite beams, J. Sound Vib. 11 (1970) $181-197$.

[2] D. Mead, Wave propagation and natural modes in periodic systems: I. mono-coupled systems, J. Sound Vib. 40 (1975) $1-18$.

[3] D. Mead, Wave propagation and natural modes in periodic systems: II. multi-coupled systems, with and without damping, J. Sound Vib. 40 (1975) 19-39.

[4] D. Mead, Wave propagation in continuous periodic structures: research contributions from southampton, 1964-1995, J. Sound Vib. 190 (1996) 495-524.

[5] M. Rumerman, Vibration and wave propagation in ribbed plates, J. Acoust. Soc. Am. 57 (1975) $370-373$.

[6] B. Mace, Periodically stiffened fluid-loaded plates, I : Response to convected harmonic pressure and free wave propagation, J. Sound Vib. 73 (1980) 473-486.

[7] B. Mace, Periodically stiffened fluid-loaded plates, II : response to line and point forces, J. Sound Vib. 73 (1980) $487-504$.

[8] D. J. Mead, Plates with regular stiffening in acoustic media: vibration and radiation, J. Acoust. Soc. Am. 88 (1990) $391-401$.

[9] C. Hodges, J. Power, J. Woodhouse, The low frequency vibration of a ribbed cylinder, part 1: Theory, J. Sound Vib. 101 (1985) 219-235.

[10] C. Hodges, J. Power, J. Woodhouse, The low frequency vibration of a ribbed cylinder, part 2: Observations and interpretation, J. Sound Vib. 101 (1985) 237-256

[11] D. Mead, N. Bardell, Free vibration of a thin cylindrical shell with periodic circumferential stiffeners, J. Sound Vib. 115 (1987) 499-520.

[12] C. B. Burroughs, Acoustic radiation from fluid-loaded infinite circular cylinders with doubly periodic ring supports, J. Acoust. Soc. Am. 75 (1984) 715-722.

[13] J. Yan, T. Li, T. Liu, J. Liu, Characteristics of the vibrational power flow propagation in a submerged periodic ringstiffened cylindrical shell, Appl. Acoust. 67 (2006) 550-569.

[14] X. Cao, H. Hua, C. Ma, Acoustic radiation from shear deformable stiffened laminated cylindrical shells, J. Sound Vib. 331 (2012) 651-670.

[15] L. Maxit, Scattering model of a cylindrical shell with internal axisymmetric frames by using the circumferential admittance approach, Appl. Acoust. 80 (2014) 10-22.

[16] Y. Tong, J. Fan, B. Wang, W. Tang, Vibroacoustic behavior of an infinitely long cylindrical shell with periodic internal lengthwise ribs, J. Acoust. Soc. Am. 144 (2018) 1528-1538. 
[17] L. Maxit, O. Guasch, V. Meyer, M. Karimi, Noise radiated from a periodically stiffened cylindrical shell excited by a turbulent boundary layer, J. Sound Vib. 466 (2020) 115016.

[18] E. Manconi, B. R. Mace, Wave characterization of cylindrical and curved panels using a finite element method, J. Acoust. Soc. Am. 125 (2009) 154-163.

[19] M. Mironov, Propagation of a flexural wave in a plate whose thickness decreases smoothly to zero in a finite interval, Sov. Phys. Acoust. 34 (1988) 318-319.

[20] V. Krylov, F. Tilman, Acoustic black holes for flexural waves as effective vibration dampers, J. Sound Vib. 274 (2004) 605-619.

[21] L. Tang, L. Cheng, Enhanced acoustic black hole effect in beams with a modified thickness profile and extended platform, J. Sound Vib. 391 (2017) 116-126.

[22] P. Zeng, L. Zheng, J. Deng, A. Elsabbagh, S. Xiang, T. Yan, Y. Wu, Flexural wave concentration in tapered cylindrical beams and wedge-like rectangular beams with power-law thickness, J. Sound Vib. 452 (2019) 82-96.

[23] V. Denis, A. Pelat, C. Touzé, F. Gautier, Improvement of the acoustic black hole effect by using energy transfer due to geometric nonlinearity, Int. J. Non Linear Mech. 94 (2017) 134-145.

[24] J. Y. Lee, W. Jeon, Vibration damping using a spiral acoustic black hole, J. Acoust. Soc. Am. 141 (2017) $1437-1445$.

[25] S. Park, M. Kim, W. Jeon, Experimental validation of vibration damping using an archimedean spiral acoustic black hole, J. Sound Vib. 459 (2019) 114838.

[26] J. Deng, L. Zheng, P. Zeng, Y. Zuo, O. Guasch, Passive constrained viscoelastic layers to improve the efficiency of truncated acoustic black holes in beams, Mech. Syst. Signal Pr. 118 (2019) 461-476.

[27] M. Ouisse, D. Renault, P. Butaud, E. Sadoulet-Reboul, Damping control for improvement of acoustic black hole effect, J. Sound Vib., Accepted (2019).

[28] S. Conlon, J. Fahnline, F. Semperlotti, Numerical analysis of the vibroacoustic properties of plates with embedded grids of acoustic black holes, J. Acoust. Soc. Am. 137 (2015) 447-457.

[29] P. A. Feurtado, S. C. Conlon, Transmission loss of plates with embedded acoustic black holes, J. Acoust. Soc. Am. 142 (2017) 1390-1398.

[30] J. Deng, L. Zheng, O. Guasch, H. Wu, P. Zeng, Y. Zuo, Gaussian expansion for the vibration analysis of plates with multiple acoustic black holes indentations, Mech. Syst. Signal Pr. 131 (2019) 317-334.

[31] W. Huang, H. Ji, J. Qiu, L. Cheng, Analysis of ray trajectories of flexural waves propagating over generalized acoustic black hole indentations, J. Sound Vib. 417 (2018) 216-226.

[32] X. Wang, H. Ji, J. Qiu, L. Cheng, Wavenumber domain analyses of vibro-acoustic decoupling and noise attenuation in a plate-cavity system enclosed by an acoustic black hole plate, J. Acoust. Soc. Am. 146 (2019) $72-84$.

[33] H. Ji, X. Wang, J. Qiu, L. Cheng, Y. Wu, C. Zhang, Noise reduction inside a cavity coupled to a flexible plate with embedded 2-d acoustic black holes, J. Sound Vib. 455 (2019) 324-338.

[34] D. O'Boy, V. Krylov, Vibration of a rectangular plate with a central power-law profiled groove by the rayleigh-ritz method, Appl. Acoust. 104 (2016) 24-32.

[35] L. Ma, S. Zhang, L. Cheng, A 2D Daubechies wavelet model on the vibration of rectangular plates containing strip indentations with a parabolic thickness profile, J. Sound Vib. 429 (2018) 130-146.

[36] V. Georgiev, J. Cuenca, F. Gautier, L. Simon, V. Krylov, Damping of structural vibrations in beams and elliptical plates using the acoustic black hole effect, J. Sound Vib. 330 (2011) 2497-2508.

[37] J. Deng, O. Guasch, L. Zheng, Ring-shaped acoustic black holes to isolate vibration in plates, J. Sound Vib. 458 (2019) $109-122$.

[38] J. Deng, O. Guasch, L. Zheng, A semi-analytical method for characterizing vibrations in circular beams with embedded acoustic black holes, J. Sound Vib. 476 (2020) 115307.

[39] J. Deng, O. Guasch, L. Zheng, Annular acoustic black holes to reduce propagative Bloch-Floquet flexural waves in periodically supported cylindrical shells, INTER-NOISE and NOISE-CON Congress and Conference Proceedings, InterNoise2019, June 16-19, Madrid, (Spain). (2019).

[40] L. Tang, L. Cheng, Broadband locally resonant band gaps in periodic beam structures with embedded acoustic black holes, J. Appl. Phys. 121 (2017) 194901.

[41] H. Zhu, F. Semperlotti, Two-dimensional structure-embedded acoustic lenses based on periodic acoustic black holes, J. Appl. Phys. 122 (2017) 065104.

[42] N. Gao, Z. Wei, R. Zhang, H. Hou, Low-frequency elastic wave attenuation in a composite acoustic black hole beam, Appl. Acoust. 154 (2019) 68-76.

[43] L. Tang, L. Cheng, Ultrawide band gaps in beams with double-leaf acoustic black hole indentations, J. Acoust. Soc. Am. 142 (2017) 2802-2807.

[44] N. Gao, Z. Wei, H. Hou, A. O. Krushynska, Design and experimental investigation of V-folded beams with acoustic black hole indentations, J. Acoust. Soc. Am. 145 (2019) EL79-EL83.

[45] M. Mironov, V. Pislyakov, One-dimensional acoustic waves in retarding structures with propagation velocity tending to zero, Acoust. Phys. 48 (2002) 347-352.

[46] O. Guasch, M. Arnela, P. Sánchez-Martín, Transfer matrices to characterize linear and quadratic acoustic black holes in duct terminations, J. Sound Vib. 395 (2017) 65-79.

[47] O. Guasch, P. Sánchez-Martín, D. Ghilardi, Application of the transfer matrix approximation for wave propagation in a metafluid representing an acoustic black hole duct termination, Appl. Math. Model. 77 (2020) 1881-1893.

[48] J. P. Hollkamp, F. Semperlotti, Application of fractional order operators to the simulation of ducts with acoustic black hole terminations, J. Sound Vib. 465 (2020) 115035.

[49] M. Mironov, V. Pislyakov, One-dimensional sonic black holes: Exact analytical solution and experiments, J. Sound Vib. 
$473(2020) 115223$.

[50] B. R. Mace, E. Manconi, Modelling wave propagation in two-dimensional structures using finite element analysis, J. Sound Vib. 318 (2008) 884-902.

[51] P. G. Domadiya, E. Manconi, M. Vanali, L. V. Andersen, A. Ricci, Numerical and experimental investigation of stop-bands in finite and infinite periodic one-dimensional structures, J. Vib. Control 22 (2016) 920-931.

[52] E. Manconi, B. R. Mace, Estimation of the loss factor of viscoelastic laminated panels from finite element analysis, J. Sound Vib. 329 (2010) 3928-3939.

[53] E. Manconi, B. R. Mace, R. Garziera, The loss-factor of pre-stressed laminated curved panels and cylinders using a wave and finite element method, J. Sound Vib. 332 (2013) 1704-1711.

[54] Y. Yang, B. R. Mace, M. J. Kingan, Vibroacoustic analysis of periodic structures using a wave and finite element method, J. Sound Vib. 457 (2019) 333-353.

[55] C. Wang, J. Lai, Prediction of natural frequencies of finite length circular cylindrical shells, Appl. Acoust. 59 (2000) $385-400$.

[56] W. Scedel, A new frequency formula for closed circular cylindrical shells for a large variety of boundary conditions, J. Sound Vib. 70 (1980) 309-317.

[57] L. Tang, L. Cheng, H. Ji, J. Qiu, Characterization of acoustic black hole effect using a one-dimensional fully-coupled and wavelet-decomposed semi-analytical model, J. Sound Vib. 374 (2016) 172-184. 